\title{
Cyber-Physical Systems: A Multi-Criteria Assessment for Internet-of-Things (IoT) Systems
}

\author{
Edgar M. Silva a and Ricardo Jardim-Goncalves ${ }^{\mathrm{a}, \mathrm{b}}$

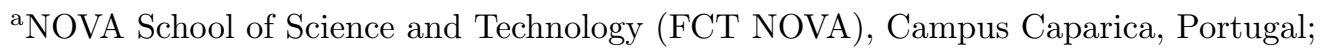 \\ ${ }^{\mathrm{b}}$ Centre of Technology and Systems (CTS), UNINOVA, Caparica, Portugal
}

\author{
ARTICLE HISTORY \\ Compiled August 2, 2019
}

\begin{abstract}
This paper addresses a multi-criteria decision problem regarding the more suitable device (system) to perform a certain task for Cyber-Physical Systems (CPS). New embedded systems provided everyday by manufacturers makes the decision on which device best fulfil an objective a very difficult work for engineers and developers. The proposed framework establishes a set of components that goes from formally describe possible solutions, criteria, constraints and priorities, capable of taking into account users' specific aspects, to finally propose a more suitable solution. To materialise all formal descriptions, a model-driven approach is followed. It allows the use and design of enablers for interoperability with standards or systems (e.g. Cyber-Physical Enterprise Systems). Models and methods are proposed to describe a device in terms of hardware, software and energy. It is enabled the use of different software languages as the integration of different, new or user-defined multi-criteria decision methods. The proposed framework shows that a better aware IoT System choice can be made and therefore stakeholders can perform a more suitable design of their Cyber-Physical Enterprise Systems.
\end{abstract}

\section{KEYWORDS}

Cyber-Physical Systems, Internet-of-Things, Model-Driven Engineering, Multi-Criteria Decision.

\section{Introduction}

A system can be defined as "a set of connected things or devices that operate together", or "a set of computer equipment and programs used together for a particular purpose" (Dictionary -). Taking into consideration a single device, it is by its own a system, composed by different hardware components interacting among them and running one or more programs/firmware. Hereupon, IoT complete system is referred as IoT Deployment or System of IoT Systems, and IoT device as IoT System or ResourceConstrained System (RCS).

This paper proposes a novel framework to assist developers in a difficult task, somehow neglected, of selecting a more suitable IoT System to execute a certain function for a Cyber-Physical Enterprise Systems. This task is normally overtaken by the selection of IoT Systems known to stakeholders, normally from lived experience or intuition. Although, it does not mean that a proper decision is being made. IoT Systems perform 
different tasks, the importance of a requisite/feature change from task to task. Make a correct, accurate decision depends many times on multiple criteria, which is a tough challenge for human beings (for Communities and Government 2009). Besides, the lack of formal descriptions to describe IoT Systems characteristics/features, capable of being used by applications in an automatic way, is also an issue. Some manufacturers provide in their websites ways to select a product from a set of choices. Products are displayed with some detailed (number of features), but limited to hardware characteristics. Interaction is completely handmade, with no way to apply user's preferences between criteria. Information data, when possible, can be collected only by user's action and change depending on the performed query. Two examples can be found in (Microchip 2019; Instruments 2019).

Literature has been more focus on functional, behaviour aspects and in activities, interactions within an IoT Deployment (Lee, Bae, and Kim 2017; Brogi and Forti 2017; Li et al. 2013) (see Section 2.1.2), rather than on IoT Systems itself. Other issues, outside this work scope, also received significant research, as big data, security, etc.

Figure 1 shows the followed research work approach. To better understand and respond to the work issues (bottom items), research was split into three objectives (identified along the path - arrow). On the left are presented literature themes used as background. The research objectives are: 1) Formally describe an IoT System; 2) characterise/analyse possible IoT System solutions; and 3) properly make a decision regarding which is the more suitable solution(s).

First, on response to IoT System, the heterogeneity nature of IoT deployments is addressed. Markets are offering a wide device diversity, with a very specific characteristic - Resource-Constrained. Methods to formally describe these imperative pieces (IoT Systems) are needed, independent of hardware platforms, as well as independent from programming languages - Platform Independent. In this way, ResourceConstrained Systems (RCS) should be study carefully and Model-Driven Engineering (MDE) techniques study and applied, since model-driven approaches revealed to be a common ground in literature (Lane and Bohn 2013; OGC 2014; W3C 2011; Agostinho 2012). Second, on analysis of IoT System solutions, addresses the characterisation of the problem that needs to be solved. As a result from the previous point, IoT Systems features are formally defined, allowing a smooth integration with other systems/tools. The problem parameterisation consists on defining the objective, criteria and constraints. Once more MDE gains here an important role. MDE tackles systems complexity through simplification and formalisation techniques during system life cycle (i.e. from design to deployment, passing by construction, operation, modification, etc.) (Agostinho 2012), and facilitates the interoperability among tools. During this phase decision makers are called to decide upon preference and qualitative criteria levels besides the normal quantitative levels. Third point addresses the decision for a suitable solution. Thematic focus on Multi-Criteria Decision-Making (MCDM), one of the most widely used decision methodologies in sciences, business, governmental and engineering worlds. MCDM methods improve decisions quality, by making the decision-making process more explicit, rational, and efficient (Cambron and Evans 1991; Wang and Triantaphyllou 2008). 


\section{Theoretical Background}

It is essential to expose the severe heterogeneity nature of an IoT Deployment, and IoT clear growing importance in today's society, with new IoT-related technologies and IoT-centric applications launch every day (Silva and Maló 2014). In this sense, Section 2.1 addresses IoT theme, Section 2.2 the model-driven thematic, focus on approach and methods for software development and enterprises and systems interoperability (Bézivin 2001; Schmidt 2006). Section 2.3 describes MCDM methodology, focused on one MCDM sub-class, where the number of solutions/alternatives is known.

\subsection{Internet-of-Things (IoT)}

Nowadays we live in highly connected world, connected to a large number of things making us more aware of the surrounding environment, consequently more proactive and less reactive (Evans 2011). IoT smart "things" have been playing an important role and will keep doing it in up-coming years with novel and diverse business models (Stankovic 2014; Undisclosed 2017). These "things" (devices, smart objects, etc.) communicate and share information in a called Wireless Sensor Network (WSN). Playing roles as actuators, gateways and distinct autonomous sensors, either physical or virtual, forming a highly heterogeneous environment to monitor physical or environmental conditions.

Manufacturers are engaged in developing new embedded systems for different purposes, to address a new variety of application domains and services. Factor that increases even more the IoT Deployments established heterogeneous nature. This brings challenging issues that have the potential to slowdown IoT development. From a technological point of view, questions have been raised on how to fully establish devices interoperability (adaptation and autonomous behaviour), and problems concerning network aspects (unique IP addresses, scalability, etc.). From a social perspective, trust, privacy and security are important aspects and must be guaranteed. Personal information must be used accordingly and no unauthorized disclosure of information (Evans 2011; Atzori, Iera, and Morabito 2010; Miorandi et al. 2012).

\subsubsection{Challenges, Barriers and Trends}

The IoT is a dynamic network infrastructure, highly heterogeneous environment, multi-manufactures and multi-service (Silva and Maló 2014). The existence of several "things" providing different types of information (temperature, noise, GPS location, etc.), brings large and heterogeneous amount of structured or unstructured data. Consequently, a high level of interoperability needs to be reached at the communication level, as well as, at service and information levels. Crossing different platforms, but established on a common ground (Bauer et al. 2013).

Through a small review, is inferred that IoT development main issues are: scalability; standardisation, specifically security and privacy; and energy consumption. IoT heterogeneity is considered a key factor, and is also mentioned communication, big data, architectures and semantic interoperability (Evans 2011; Stankovic 2014; Undisclosed 2017; Atzori, Iera, and Morabito 2010; Miorandi et al. 2012).

One of the main drivers of the IoT investigation is the European Projects. Research programmes focus on IoT Platforms, smart systems integration, Cyber-Physical System (CPS), big data, security, etc. Addressing also innovation, interoperability for industry and services applied to large scale pilots (e.g. test-beds). 


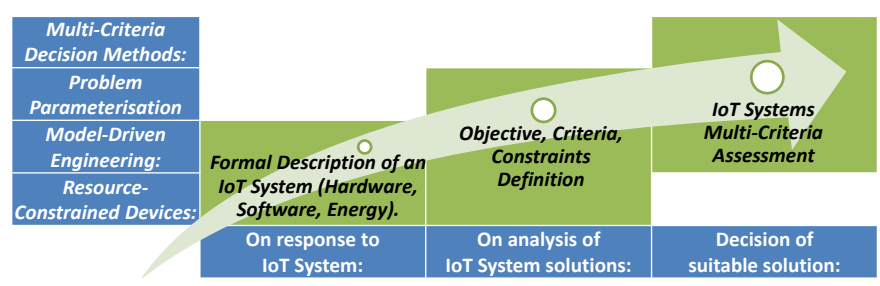

Figure 1. Research Approach: Path to Select More Suitable RCS.

Io $\mathrm{T}$ is a relevant part in the new generation of information technology, with a great potential. But it is also a challenge due to the vast range of issues that still need to be addressed.

\subsubsection{Standards}

One of the key issues in IoT is standardisation. To tackle this, many international organisations are working on defining standards at different levels.

European Telecommunications Standards Institute (ETSI) is an independent, notfor-profit organisation in technological openness and interoperability. Institute of Electrical and Electronics Engineers (IEEE) focus on bringing the IoT global technical community together, providing a platform where professionals can learn and share knowledge. Internet Engineering Task Force (IETF) is a large open international community concerned with the evolution of the Internet architecture and its smooth operation, and aims to extend the Web architecture to most constrained networks. Object Management Group (OMG) is an international, open membership, not-for-profit technology standards consortium (addressed here, are MDA, DDS and SysML). World Wide Web Consortium (W3C) is an international community working on Web standards, created a community group to easy the adoption and development of Web of Things standards. Organisation for the Advancement of Structured Information Standards (OASIS) is a global not-for-profit consortium, focus on IoT, security, energy, etc.

Dozens other organisations and coalitions have been formed, to unify the fragmented, fractured and organic IoT landscape.

Wireless communication protocols standard list is wide. Figure 2 shows a few examples. Commonly communication protocols are divided in terms of transmission range. Very close transmission, Wireless Personal Area Network (WPAN), includes NearField Communication (NFC), Zigbee and Bluetooth protocols. Covering an area up to 1 kilometres, Wireless Local Area Network (WLAN), is found Wi-Fi protocol, a technology based on IEEE 802.11 standards, with different radio bands being $2.4 \mathrm{GHz}$ and $5 \mathrm{GHz}$ the most common. For transmissions up to 50 kilometres, Wireless Metropolitan Area Network (WMAN), protocols such as SigFox, LoRa or NBIoT can be used. Sigfox and LoRa (Long-Range) use Industrial, Scientific, and Medical (ISM) bands, while NB-IoT is a cellular IoT technology working in a licensed spectrum (Long-Term Evolution (LTE), GSM and UMTS bands). NB-IoT, also known as LTE Cat NB1, is a part of the $3 \mathrm{GPP}$ Release 13 standard. Covering higher transmissions range, Wireless Wide Area Network (WWAN), GPS is an example, owned by the United States government, transmits radio signals for position, navigation and time services. Zigbee, Sigfox and LoRa operate on different frequencies depending on the country.

Data exchange protocols is another area related to communication. Data elements are exchange to trigger a certain procedure or just to transfer data. Messages between 


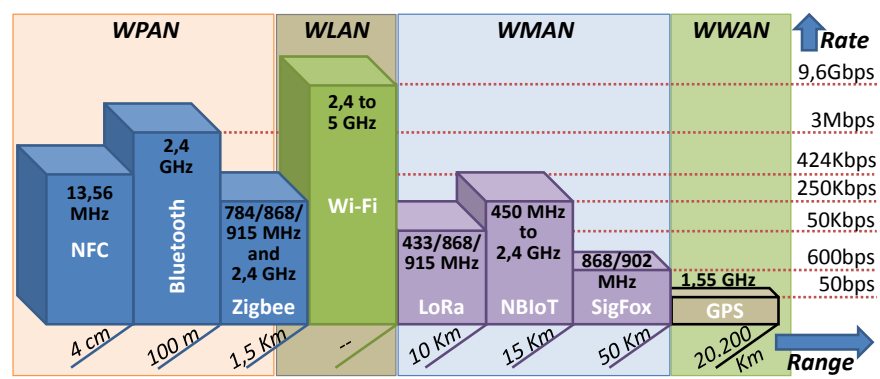

Figure 2. Wireless Protocols: Transfer Range, Rate \& Frequency.

nodes/components are based in mechanisms often called Message-Oriented Middleware (MOM), a specific software class used in distributed environments (Luzuriaga et al. 2015). Advanced Message Queuing Protocol (AMQP) is built upon two main models, the transport and queuing model (O'Hara 2007). The transport model is based on a binary protocol and the queuing model deals with storage and services semantics to achieve interoperability among entities. Message Queuing Telemetry Transport (MQTT) (Banks and Gupta 2014), as AMQT, is a standard supported by the OASIS organisation. It is a Machine-to-Machine (M2M) (or Client Server) Publish-Subscribe (Pub-Sub) message protocol, built to be simple, open, lightweight and easy to implement a connection. Constrained Application Protocol (CoAP) (Shelby, Hartke, and Bormann 2014), specified as RFC 7252 by IETF, was designed for M2M applications, following a request/response model, with capability to discover services and resources, focus on specific characteristics of constrained environments (nodes and networks). Data Distribution Service (DDS) (Pardo-Castellote 2003), is a Pub-Sub standard for real-time systems, managed by the OMG. DDS is a complete decentralised architecture, providing a dynamic discovery service, Quality-of-Service (QoS), security, among others characteristics. Regarding the connectivity space they address, CoAP is focus on Inter Device and Device - Gateway communication. AMQP focus on GatewayCloud communication, while MQTT and DDS cover all scope, i.e. Device-Cloud communication.

Standards have also been proposed to define systems, modelling requirements, behaviours, processes, etc., supporting interoperability at a syntactic as semantic level. One example is OMG' Systems Modelling Language (SysML) (Lane and Bohn 2013), a general-purpose architecture modelling language for engineering systems. SysML is an extension of OMG's Unified Modeling Language (UML), designed to support the specification of requirements, structure and behaviour, as verification and engineering systems validation. Another is Sensor Model Language (SensorML) (OGC 2014) an OGC approved standard. The main objective is to enable interoperability, syntactic as semantic using ontologies and semantic mediation. SensorML represent components, physical (e.g. detectors, actuators) and non-physical (e.g. mathematical operations or functions) as processes. SensorML describes sensors functional models, although it can provide detailed information of a sensor hardware design. Other example is the W3C Semantic Sensor Network (SSN) (W3C 2011) an ontology to describe sensors and observations. SSN was developed with two main objectives. Create ontologies to describe sensors, and provide an extension (semantic annotations) to the SensorML. 


\section{2. $\quad$ Model-Driven Engineering}

Over the past six decades, researchers and developers have been trying to create high level software abstractions for applications development and for systems design-phase. These abstractions focus on language as in platform technologies (Schmidt 2006). From the efforts made, is important to emphasize in 1980s the Computer-Aided Software Engineering (CASE) and 2000s the Model-Driven Engineering (MDE).

CASE is a computer-assisted method to organise, control software development on large and complex projects, involving many people and project parts. One of CASE goals is to enable a more accurate and a better analysis of software programs (Schmidt 2006; Case 1985). Increasing organisations productivity, decrease costs, improve project controls and products quality.

Although, CASE attracted considerable attention in research community, it was not widely adopted, since graphical representation languages were mapped poorly to languages and main platforms. Lack of Quality-of-Service (QoS) properties (e.g. fault tolerance and security), lead to large amounts of code to compensate (Schmidt 2006). Also, the inadequate standardisation, high expectations (rapid payback), and inappropriate implementations (applied for the first time on critical or shorten deadline projects) were associated issues/risks.

Nowadays, IT managers, entrepreneurs and software developers use models for almost anything. The constant increase of systems complexity, shorter development cycles and high quality expectations, has push forward, the use of model-driven techniques on modelling/design critical stages of systems development. With the use of model-driven approaches, models have become primary artefacts in software development (Hutchinson, Rouncefield, and Whittle 2011).

This trend, Model-Driven, has been followed by academia and industry regarding not only automatic software development, but also enterprises integration and interoperability.

With the promising software engineering approach, MDE, comes the buzz words - Model and Meta-Model. A basic principle followed along the years, states that "everything is an object". MDE has a similar principle, which is "everything is a model" (i.e. entities, software, services, platforms, etc.) (Bézivin 2005). A model can be seen as a set of statements regarding a System Under Study (SUS) or a class of the SUS. Meta-Model is a specification model, it specifies what statements can be made within the Model.

\subsubsection{Model-Driven Architecture (MDA)}

Model-Driven Architecture (MDA) (Miller et al. 2003) was initially design for software development, although it has been used in other areas like Business Process Modelling and systems/enterprises interoperability. MDA presents three abstractions layers, Computation Independent Model (CIM), Platform Independent Model (PIM) and Platform Specific Model (PSM), to cover systems development process (Miller et al. 2003; Singh and Sood 2009). Supported by two types of transformations, horizontal and vertical, in which a variety of possible transformation categories can be established, such as, Marking, Meta-Model transformation, Model transformation or Model Merging (Singh and Sood 2009). These transformations assist on the development process (more automatic) of new system functionalities, and can also be used to perform systems/enterprises interoperability by matching process phases or data descriptions from one company/system to another. Although, MDA does not specify 
transformations from CIM to PIM level since it uses an OMG standard, the MetaObject Framework (MOF) that provides basis for a meta-model definition.

\subsection{Multi-Criteria Decision-Making (MCDM)}

Decisions are something that all people have to face daily. Questions, simple or complex, are placed constantly and when the number of factors increases, more difficult it is to make a correct and accurate decision for human beings. MCDM is one of the most widely used decision methodologies in sciences, business, governmental and engineering worlds. It is a process capable of analyse possible solutions based on a set of different criteria, and identify one or more suitable solutions to be applied (for Communities and Government 2009; Cambron and Evans 1991; Wang and Triantaphyllou 2008). Next, methods are presented focus on problems with a finite and known number of alternatives.

\subsubsection{Multi-Criteria Decision-Making (MCDM) Methods}

MCDM methods have been applied to engineering problems, providing useful insights to decision makers, making their decisions more qualified to overcome complex problems (Kolios et al. 2016). Based on (Kolios et al. 2016; Sabaei, Erkoyuncu, and Roy 2015; Aruldoss, Lakshmi, and Venkatesan 2013), three of the most widely used decision making methods are the AHP, PROMETHEE and ELECTRE.

Analytic Hierarchy Process (AHP) (Saaty 1977), objective is to solve problems with multiple and conflicting criteria. AHP is a powerful decision making methodology that defines solutions ranking through a pair-wise comparison of multiple criteria. AHP original methodology has 4 steps (Figure 3a). First step is to decompose a complex problem into a systematic hierarchy structure (Aldlaigan and Buttle 2002). At the top is place the objective(s), followed by assessment criteria considered important, and at the bottom the solutions/alternatives. Second step, applies a criteria pairwise comparison based on decision maker judgement, creating a Saaty 1-9 scale to assess criteria priority. Step three, consists on classify solutions individually. Individual score is obtained through an analysis of each criteria contribution to the problem objective. Then a prioritization theory is followed. Although, AHP presents some disadvantages, such as pairwise comparison is based on decision makers' subjective judgement and criterion weight has a direct impact on the final score.

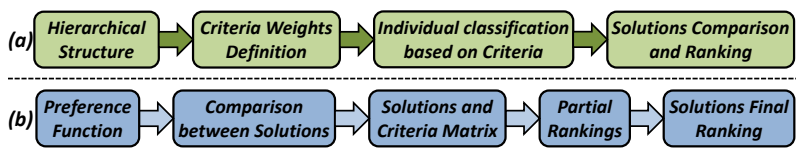

Figure 3. a) Original AHP Methodology; b) PROMETHEE Methodology.

PROMETHEE (Preference Ranking Organization Method for Enrichment Evaluation) (Brans and Vincke 1985) presents 6 different versions based on ranking. PROMETHEE I, consists on partial ranking; version II on complete ranking; version III performs ranking based on intervals and PROMETHEE IV is for the continuous case; version $\mathrm{V}$ has integer linear programming and net flows; and finally the PROMETHEE VI includes a representation of the human brain (José Figueira 2005; Mela, Tiainen, and Heinisuo 2012). The PROMETHEE methodology can be described in 5 steps (Figure $3 \mathrm{~b}$ ). Decision maker chooses a preference function (e.g. 
Boolean function), and compare solutions. Comparison results and criteria values are used to form a matrix, on which PROMETHEE versions are applied. This method is consistent, easy to use and does not need great interaction with decision makers. Although, presents as downside the incapability to react when a new alternative is introduced.

Elimination and Choice Expressing the Reality (ELECTRE) method (Roy 1991) (with six variations) is a preference-based model, performing pair-wise comparison between solutions, with 3 types of relations: is preferred to; is indifferent to; and incomparable to. All methods are based on the same background, but have different processes. In ELECTRE I and IS, a single solution or a group of solutions are selected and assigned as possible solution. ELECTRE II was developed to deal with problems that need to rank solutions from best to the worst. ELECTRE III introduced pseudocriteria and fuzzy binary outranking relations. ELECTRE Iv to rank solutions without the use of relative criteria coefficients (the only method that do not use it). Finally, ELECTRE TRI assigns categories to solutions (Kolios et al. 2016; Brans and Vincke 1985). ELECTRE do not frequently lead to one solution standing out from the others. For this reason the method is considered to be more suitable for problems with several solutions and not so many criteria.

\section{A Resource-Constrained Systems (RCS) Multi-Criteria Assessment}

This section presents the conceptual solution (Figure 4) to perform a careful assessment of IoT Systems. The principle is to go from a set of data (alternatives), through the definition of a purpose/objective (problem to solve), to a more conscious decision of which is the proper solution for a certain problem.

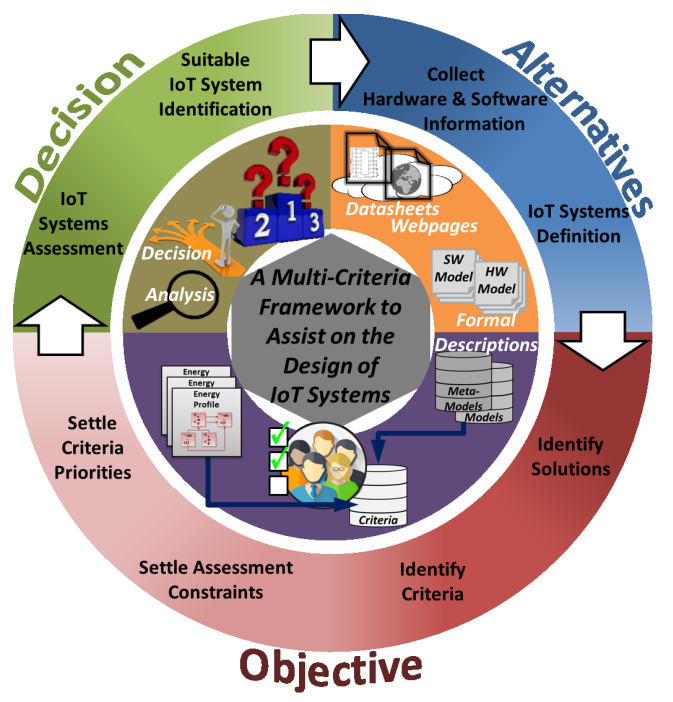

Figure 4. A RCS Multi-Criteria Assessment: Framework.

First, identification of alternatives through research of webpages, datasheets, etc., collecting hardware, software and general information to create/generate formal descriptions based on established models to serve as RCS definitions. Accomplishing this, is then possible formalise an objective. Identify what solutions are to be considered, criteria to be analysed and criteria constraints and priorities. Finally, is possible 
to apply methods to identify/decide on which is/are most suitable IoT System(s) to perform the defined task within a Cyber-Physical Enterprise System.

\subsection{RCS Multi-Criteria Assessment: Functional Architecture}

To materialise the high level abstraction structure of the framework (Figure 4) an architecture is presented in Figure 5, based upon 6 main blocks.

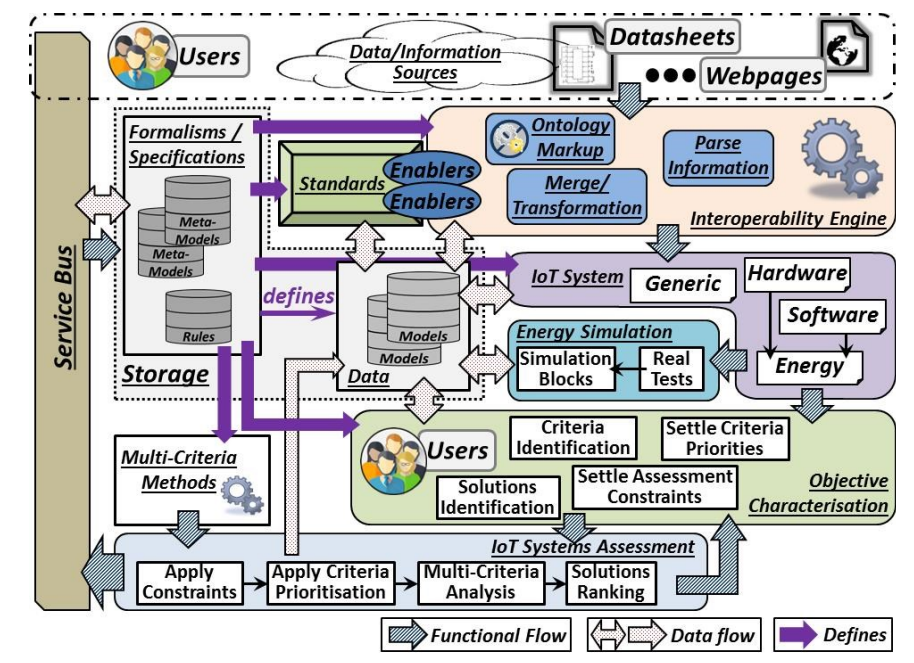

Figure 5. A RCS Multi-Criteria Assessment: Architecture.

Interoperability Engine is responsible for mapping the information retrieved from data/information sources to the IoT System specifications. This block takes use of the MDA techniques to fulfil its goal. Enablers can be design to import/export data from other specifications (standards or not), enabling interoperability with other systems or tools. Ontologies can be used to identify and reasoning over data retrieved, for example, matching (e.g. units' conversion) can be accomplished with semantic analysis. Storage, divided in two sections: "Formalisms/Specification", serves as repository for specification models, standards specifications and rules that execute data interchange between models; "Data", for data models that are created and consequently have to be stored along the architecture functional process. IoT System, a full representation of an IoT System, following the established specifications and relations. An IoT System, if it does not exist in the Storage block, can be defined from already available specifications (e.g.: combine different hardware parts). It can also be settle a software program, energy profile and the possibility to specify other important features related to the IoT System being created. This information serves as input for Energy Simulation or Objective Characterisation blocks. Energy Simulation, responsible for energy consumption simulation, through IoT Systems specifications and available data from real life tests. Objective Characterisation, is the problem to solve (a purpose/objective) definition. It is composed by four operations. Solutions Identification - for each objective different solutions can be identified and some excluded from the original group; Criteria Identification - decision makers choose the important features to be analysed; Assessment Constraints - decision makers indicate criteria restrictions, such as optimization type, availability and set thresholds; and finally, Criteria Priorities decision makers determine which criterion/feature is more important regarding the others (preference levels). IoT Systems Assessment, produces a result, propose 
the more suitable solution for the defined problem. Constraints and selected MCDM methods (existing or user defined) are applied. In case of assessment outcome not satisfactory for some reason (e.g. applied constraints excluded all solutions), is possible to go back for adjustments (e.g. criteria, constraints). Qualitative and quantitative analysis is available.

Data/Information Sources and Service Bus are representative blocks of available components and not core/contribution of the proposed architecture. Therefore, not address here.

\subsection{RCS Multi-Criteria Assessment: Specification Models}

Figure 6 depicts the main specification models, which defines and ensures relationship within the proposed framework and enables interoperability with other information models. Five packages were created to formally describe all aspects in the assessment of IoT Systems. The work developed in (Undisclosed 2017, 2015) are previous and standalone versions of MCAM (Multi-Criteria Analysis Model) and RCSH (ResourceConstrained System Hardware) models, respectively.

IoTSAG (IoT Systems Analysis Generic) specification is used to instantiate properties/criteria and related aspects (units and domain units), maintaining interoperability and consistency among framework models and tools.

MCAM now uses IoTSAG specifications. Provides means to instantiate outcomes, and the most relevant change is a new interface class for specification of new/different/user defined MCDM methods within the same problem (multi assessments by changing criteria, constraints or MCDM methods).

RCSH model now uses property instantiation from IoTSAG. It is one core part of an IoT System definition.

RCSM (Resource-Constrained System Model) is represented by hardware characteristics (RCSH model), by a software language (program) and if available by energy information. An interface class (SoftwareModel) allow instantiation of different software languages to describe the program used. Different energy profile models can be used, enabled by using the same approach (interface class). The RCSM enables features instantiation, through IoTSAG properties, such as cost, memory used, final physical size, etc., which are directly connected to a RCS in its final form and not to one of the parts that builds it. A device (physical part) can be associated with different software languages, forming different RCSs. Or simply by joining different hardware parts (shields/expand boards that can be coupled together). That is, from one RCSH or one software model multiple RCSs (multiple solutions) can be obtained.

The final model, working as glue, is the IoTSACM (IoT Systems Analysis Core Model). Through this specification model is possible to define/reference known RCS (possible solutions), specify objectives, criteria and constrains (definition of tasks to be performed), and select/apply different MCDM methods, even for the same goal. Consequently, a complete formalisation is provided, specifying how to perform a more aware, proper decision regarding the selection of an IoT Systems for each task that needs to be executed in an entire IoT Deployment.

MCAM and RCSM present the main feature of allowing different (even new) decision methods, programming languages and energy profiles. In these cases, the proposed framework is not bound to restrict, pre-established specification models. However, these models must respect two rules. Properties must be specified by IoTSAG model and main model object has to inherit the corresponding interface (e.g. SoftwareModel). 


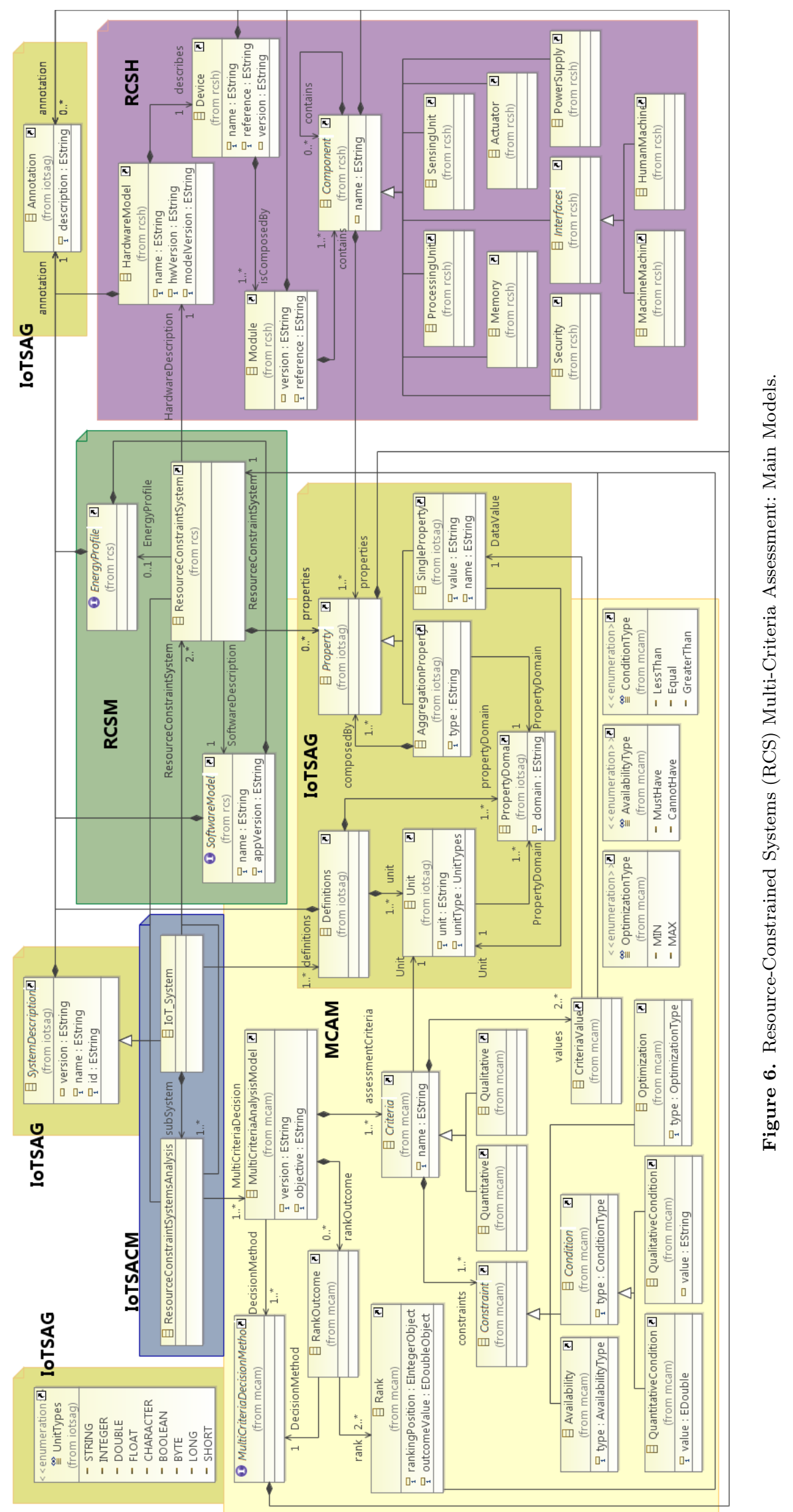




\section{Materials and Methods}

Figure 7(a) presents a specification model for AHP decision method. It enables the definition of criteria priority relation, "priorityValue", i.e. criteria $a$ is more important than criteria $b$. Comparison can use different scales, by default uses Saaty 1-9 scale. Consistency ratio is by default $10 \%$, i.e. value used to analyse correctness of priorities assigned by decision makers. On the other hand, Figure 7(b) depicts a specification model for ELECTRE decision method. Also in this case, the core model class, "ELECTREModel", inherits from the corresponding interface, MultiCriteriaDecisionMethod (MCAM). Criteria weights, concordance and nonconcordance thresholds are defined, as ELECTRE method refers it. Both models reference Criteria from MCAM. Figure 8 shows a XMI instantiation example (partial) of AHP proposed model. Criterion Implementation Time ("@assessmentCriteria.1") was considered by stakeholders, one third less important than criterion Energy ("@assessmentCriteria.0"). Data retrieved from (Undisclosed 2017).

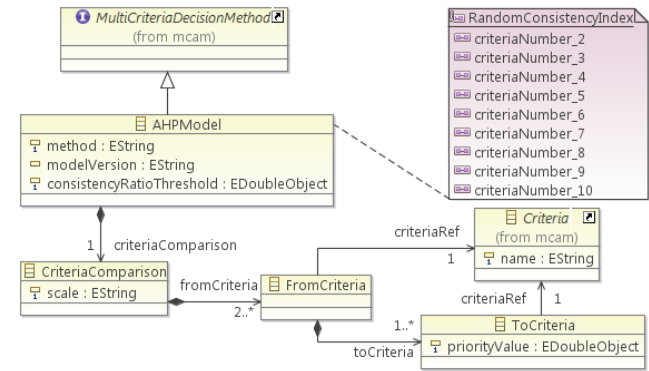

(a) AHP

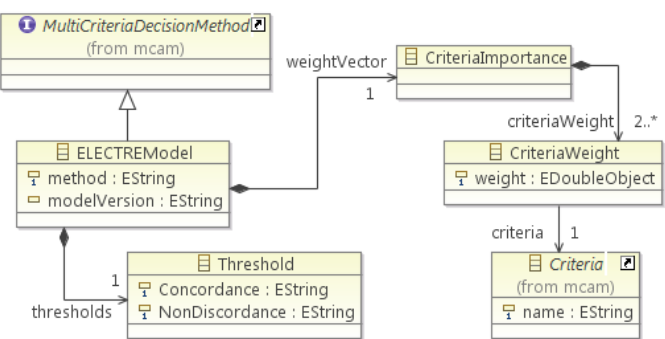

(b) ELECTRE.

Figure 7. MCDM Methods: Proposed Meta-Models.

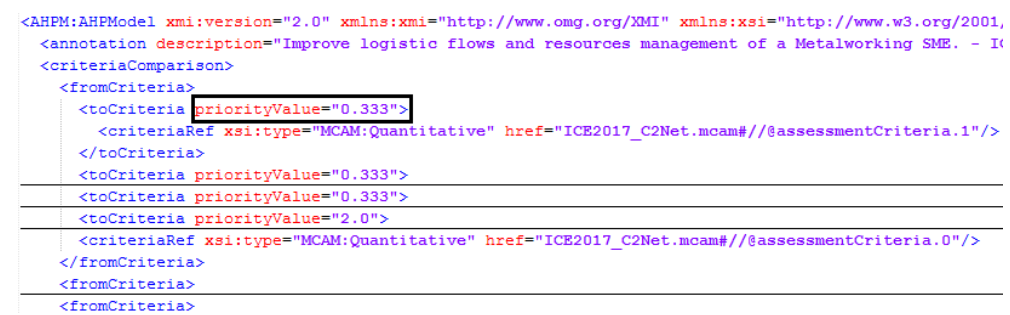

Figure 8. XMI instantiation of AHP model.

Table 1. Interoperability: SensorML Mapping Example.

\begin{tabular}{|c|c|c|}
\hline Source Package: & Source Class: & Target Class: \\
\hline IoTSACM & ResourceConstrainedSystemAnalysis & PhysicalSystem \\
\hline RCSM & ResourceConstrainedSystem & PhysicalComponent \\
\hline IoTSAG & Property; Unit & InputList; OutputList \\
\hline
\end{tabular}

A framework feature is the high interoperability with other systems or models (e.g. standards). As an example, Table 4 shows classes' matching between proposed models (Source) and standard SensorML (Target). Figure 9(a) depicts how such process is obtained using MDA techniques. In PSM layer, data is converted from one model to another. PIM layer, responsible for models specification (meta-models) and transformation rules definition. Top layer, CIM, offers meta-model definition (see Section 
2.2.1). Figure 9(b) presents the implementation using Atlas Transformation Language (ATL).

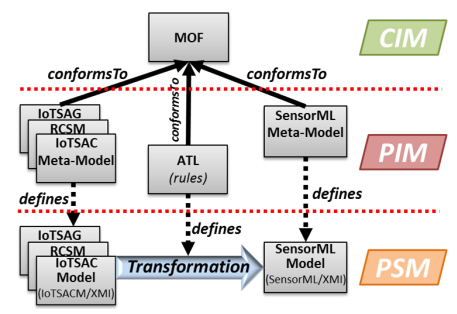

(a) Models Mapping.

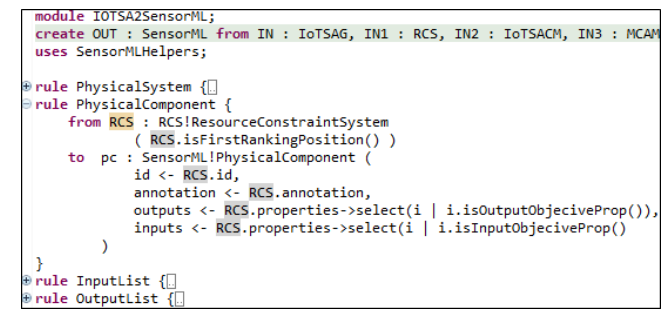

(b) ATL Transformation Code.

Figure 9. Interoperability with SensorML Standard.

\section{Verification in Industrial Environment}

IoT Systems assessment methodology has been applied to an industrial scenario C2Net Portuguese pilot (see (Undisclosed 2017) for complete implementation details). Stakeholders were able to perform a conscious decision regarding the more suitable solution for a specific task during the design phase of the IoT deployment. The objective was to select a suitable device to act as C2Net Sensor (see Figure 10), taking into consideration energy consumption; implementation time; built/adapt hardware; cost; and solution processing speed. Possible solutions were based on Arduino platforms, communication CAN, and different approaches to build the solution.

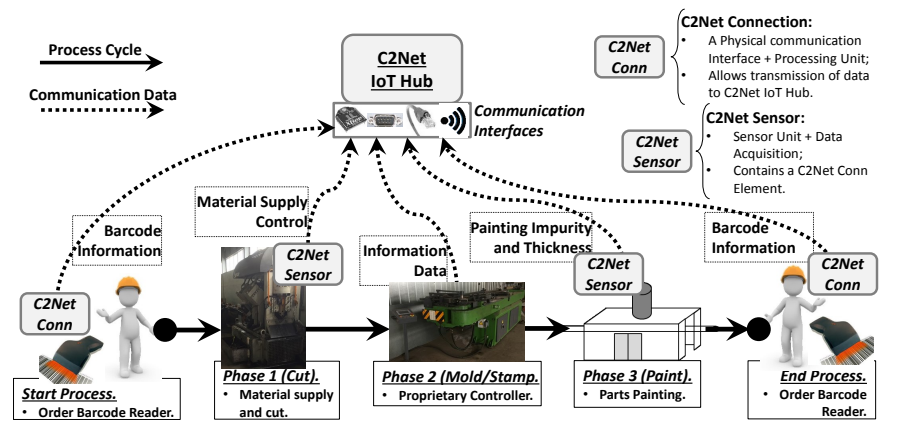

Figure 10. Industrial Scenario: C2Net pilot (retrieved from (Undisclosed 2017)).

\section{Results \& Discussion}

Figures 7(a) and 7(b) are examples of an important feature provided by the framework - openness to different specifications. Users can apply their methods, providing specification models and implementation enablers. Same methodology is applied for software languages and energy profiles. Figure 9(a) demonstrates means to achieve interoperability, either with company tools (proprietary) or, as in the example, standards.

Review on available standards and only on a specific area, showed that alternatives (e.g. different communication range) are vast and can pose as important decision 
criteria for stakeholders. Consequently, it is imperative the use of tools or methods to assist engineers on the decision process.

This work does not try to solve IoT heterogeneous nature, but rather assist stakeholders in their decisions regarding the proper IoT System to perform a certain task, from a large set of possible solutions and criteria. Computation of IoT System assessment was presented in (Undisclosed 2017) through an industrial validation. The industrial scenario, from the C2Net European Project, involved a Metalworking SME with the objective of improving management of logistic flows and resources. Methodology was applied to select the more suitable IoT Systems to act as machinery sensor nodes (data acquisition).

Necessary adjustments were made to integrate the standalone versions of MCAM (Undisclosed 2017) and RCSH (Undisclosed 2015) previously presented, with the proposed IoT System selection framework.

\section{Conclusions \& Final Contributions}

This work tackles the process of perform an aware choice regarding the more suitable IoT System to execute a certain task, where different criteria must be analysed. It is proposed a complete framework to achieve the research objectives. Models and methods were presented to formally describe an IoT System (hardware, software and energy). Specification to characterise/analyse possible IoT System solutions is presented and MCDM methods can be applied to properly make a decision. Highlighting the use of different software languages and MCDM methods, possible inclusion of energy consumption aspects and the use/design of enablers for Cyber-Physical Enterprise Systems and standards interoperability. All integrated in one very useful tool.

Specification models for AHP and ELECTRE MCDM methods were also presented.

As future work is foreseen creation of methods to gather automatically RCS information. For this is predicted the use of ontologies to allow identification and reasoning over important RCS features, and consequently fill the framework models. Furthermore, the integration with European Project vf-OS (Virtual Factory Operating System) responsible for providing key system resources and a set of specific services for manufacturing.

\section{Acknowledgement(s)}

This research work was partially supported by funds provided by the European Commission in the scope of FoF/H2020-636909 C2NET, FoF/H2020-723710 vf-OS and ICT/H2020-825631 ZDMP.

\section{References}

\section{References}

Agostinho, Carlos. 2012. "Sustainability of systems interoperability in dynamic business networks." PhD diss., Faculdade de Ciências e Tecnologia da Universidade Nova de Lisboa, Caparica.

Aldlaigan, Abdullah H., and Francis A. Buttle. 2002. "SYSTRA-SQ: a new measure of bank service quality." Int. J. of Service Ind. Manag. 13 (4): 362-381. 
Aruldoss, Martin, T. Miranda Lakshmi, and V. Prasanna Venkatesan. 2013. "A Survey on Multi Criteria Decision Making Methods and Its Applications." American J. of Information Systems 1 (1): 31-43.

Atzori, Luigi, Antonio Iera, and Giacomo Morabito. 2010. "The Internet of Things: A Survey." Comput. Network 54 (15): 2787-2805.

Banks, Andrew, and Rahul Gupta. 2014. "MQTT Version 3.1.1." 10. http://docs.oasisopen.org/mqtt/mqtt/v3.1.1/os/mqtt-v3.1.1-os.pdf.

Bauer, Martin, Mathieu Boussard, Nicola Bui, Francois Carrez, Christine Jardak, Jourik De Loof, et al. 2013. "Deliverable D1.5 - Final architectural reference model for the IoT v3.0." 7. FP7-257521 IoT-A "The Internet of Things - Architecture".

Bézivin, Jean. 2001. "From Object Composition to Model Transformation with the MDA." In Proc. of the 39th Int. Conference and Exhibition on Technol. of Object-Oriented Languages and Systems (TOOLS39), TOOLS '01, Washington, DC, USA, 8, 350-. IEEE Comput. Soc.

Bézivin, Jean. 2005. "On the Unification Power of Models." Software and Systems Modeling (SoSyM) 4 (2): 171-188.

Brans, Jean Pierre, and Philippe Vincke. 1985. "Note-A Preference Ranking Organisation Method." Manag. Science 31 (6): 647-656.

Brogi, A., and S. Forti. 2017. "QoS-Aware Deployment of IoT Applications Through the Fog." IEEE Internet of Things J. 4 (5): 1185-1192.

Cambron, Kenneth E., and Gerald W. Evans. 1991. "Layout design using the analytic hierarchy process." Comput. and Industrial Eng. 20 (2): 211-229.

Case, Albert F. 1985. "Computer-aided Software Engineering (CASE): Technology for Improving Software Development Productivity." SIGMIS Database 17 (1): 35-43.

Dictionary, Cambridge. -. "system." https://dictionary.cambridge.org/dictionary/english/system.

Evans, Dave. 2011. The Internet of Things: How the Next Evolution of the Internet Is Changing Everything. White paper. CISCO Internet Business Solutions Group (IBSG). http://www.cisco.com/web/about/ac79/docs/innov/IoT_IBSG_0411FINAL.pdf.

for Communities, Department, and Local Government. 2009. Multi-criteria analysis: a manual. Manual. Bressenden Place, London: Crown. http://eprints.lse.ac.uk/12761/1/Multicriteria_Analysis.pdf.

Hutchinson, John, Mark Rouncefield, and Jon Whittle. 2011. "Model-driven engineering practices in industry." In Proc. of the 33rd Int. conference on Software eng., ICSE '11, 633-642. ACM.

Instruments, Texas. 2019. "BOM \& cross reference tool." https://bomcross.ti.com/en/.

José Figueira, Matthias Ehrogott, Salvatore Greco. 2005. Multiple Criteria Decision Analysis: State of the Art Surveys. Int. series in operations research \& manag. science ed., Vol. 78. New York, USA: Springer-Verlag.

Kolios, Athanasios, Varvara Mytilinou, Estivaliz Lozano-Minguez, and Konstantinos Salonitis. 2016. "A Comparative Study of Multiple-Criteria Decision-Making Methods under Stochastic Inputs." Energies 9 (7): 566.

Lane, Jo Ann, and Tim Bohn. 2013. "Using SysML modeling to understand and evolve systems of systems." Syst. Eng. 16 (1): 87-98.

Lee, Suk Kyu, Mungyu Bae, and Hwangnam Kim. 2017. "Future of IoT Networks: A Survey." Applied Sciences 7 (10).

Li, F., M. Vögler, M. Claeßens, and S. Dustdar. 2013. "Towards Automated IoT Application Deployment by a Cloud-Based Approach." In IEEE 6th Int. Conference on Service-Oriented Computing and Applications, Dec, 61-68.

Luzuriaga, Jorge E., Miguel Perez, Pablo Boronat, Juan Carlos Cano, Carlos Calafate, and Pietro Manzoni. 2015. "Impact of mobility on Message Oriented Middleware (MOM) protocols for collaboration in transportation." In Proc. of the 19th Int. Conference on Comput. Supported Cooperative Work in Design, CSCWD '15, 5, 115-120. IEEE.

Mela, Kristo, Teemu Tiainen, and Markku Heinisuo. 2012. "Comparative study of multiple criteria decision making methods for building design." Advanced Eng. Informatics 26 (4): $716-726$. 
Microchip. 2019. "New/Popular Microcontroller and Processors Products." https://www.microchip.com/ParamChartSearch/chart.aspx?branchID=1005.

Miller, Joaquin, Jishnu Mukerji, Mariano Belaunde, Fred Cummins, Desmond Dsouza, Keith Duddy, et al. 2003. MDA Guide Version 1.0.1. Technical Report omg/2003-06-01. Needham, MA, USA: Object Management Group, Inc. http://www.omg.org/cgi-bin/doc?omg/03-0601.pdf.

Miorandi, Daniele, Sabrina Sicari, Francesco De Pellegrini, and Imrich Chlamtac. 2012. "Internet of things: Vision, applications and research challenges." Ad Hoc Networks 10 (7): $1497-1516$

OGC. 2014. "Sensor Model Language (SensorML)." 2. https://www.opengeospatial.org/standards/sensorml.

O’Hara, John. 2007. "Toward a Commodity Enterprise Middleware." Queue 5 (4): 48-55.

Pardo-Castellote, Gerardo. 2003. "OMG Data-Distribution Service: Architectural Overview." In Proc. of the 23rd Int. Conference on Distributed Computing Systems, ICDCSW '03, Washington, DC, USA, 5, 200-. IEEE Comput. Soc.

Roy, Bernard. 1991. "The outranking approach and the foundations of electre methods." Theory and Decision 31 (1): 49-73.

Saaty, Thomas L. 1977. "A scaling method for priorities in hierarchical structures." J. of Mathematical Psychology 15 (3): $234-281$.

Sabaei, Davood, John Erkoyuncu, and Rajkumar Roy. 2015. "A Review of Multi-criteria Decision Making Methods for Enhanced Maintenance Delivery." Procedia CIRP 37: 30 - 35.

Schmidt, Douglas C. 2006. "Guest Editor's Introduction: Model-Driven Engineering." IEEE Computer 39 (2): 25-31.

Shelby, Z., K. Hartke, and C. Bormann. 2014. "The Constrained Application Protocol (CoAP)." https://tools.ietf.org/html/rfc7252.

Silva, Edgar M., and Pedro Maló. 2014. "IoT Testbed Business Model." Advances in Internet of Things 4 (4): 37-45.

Singh, Yashwant, and Manu Sood. 2009. "Models and Transformations in MDA." In Proc. of the First Int. Conference on Computational Intelligence, Communication Systems and Networks, CICSYN '09, 7, 253-258. IEEE Comput. Soc.

Stankovic, John A. 2014. "Research Directions for the Internet of Things." Internet of Things J., IEEE 1 (1): 3-9.

Undisclosed. 2015. "Undisclosed for Review." In Proc. of the Technological Innovation for Cloud-Based Engineering Systems, IFIP Advances in Information and Communication Technol., 3, -. Springer Int. Publishing.

Undisclosed. 2017. "Undisclosed for Review." In Int. Conference on Engineering, Technol. and Innovation (ICE/ITMC), June, -.

W3C. 2011. "Semantic Sensor Network XG Final Report." 6. https://www.w3.org/2005/Incubator/ssn/XGR-ssn-20110628/.

Wang, Xiaoting, and Evangelos Triantaphyllou. 2008. "Ranking irregularities when evaluating alternatives by using some ELECTRE methods." Omega 36 (1): 45-63. 